\title{
A Case of Too Much Doggy Love
}

\author{
Melissa J MacPherson, PhD (Meds 2014) \\ Faculty Reviewer: Dr John Payne, MCISc, MD, CCFP, FCFP (Department of Family Medicine)
}

A

nimal bites, especially bites to the head, neck and face, are common injuries in children. ${ }^{1}$ It is estimated that in the pediatric population dog bites account for $0.3 \%$ to $1.5 \%$ of all presentations for medical care. ${ }^{1}$ Children are most likely to be bitten by a familiar dog at home and are frequently bitten due to a provoked attack. ${ }^{1-3}$ Appropriate adult supervision could prevent many of these bites from occurring.

It was while I was on a rural family medicine rotation in Dresden, a small farming community of 2800 people in Southwestern Ontario, that I gained exposure to pediatric dog bite injuries. My sweet patient, an animal lover, was playing with a neighbour's 8-week-old puppy when the injury occurred. She picked up the puppy and squeezed him too hard and the puppy bit her ear in an attempt to escape; a very natural reaction for a small animal. She presented to the local emergency department where the wound was cleaned and closed using dissolvable sutures. The local health department was contacted and informed of her dog bite injury. The following day she presented to our clinic for follow-up care in the company of her mother, her older sister and her stuffed animal - a black doggy. It was in the family medicine clinic where I had the pleasure of meeting her and her four-legged stuffed companion.

Since the puppy at fault was only 8 weeks old, he had not yet received his immunizations. Our 3-year-old patient needed to undergo rabies postexposure prophylaxis (PEP) treatment to prevent a fatal rabies infection. The unimmunized puppy lived on a farm where he theoretically could have been exposed to rabid wild animals. He was placed under quarantine and underwent testing for the rabies virus. The Center for Disease Control in the United States recommends a quarantine period of 10 days for all unimmunized dogs involved in bite injuries. ${ }^{4}$ Animals are considered to be vaccinated against the rabies virus 28 days after the initial immunization since this is when peak rabies virus antibody titer is reached. ${ }^{4}$ All dogs are required to have booster shots on a yearly basis to ensure that their immune status against the virus is maintained. ${ }^{4-5}$ Unfortunately, the puppy in our case was now in for a miserable 10 days of confinement so that he could be monitored for signs and symptoms of rabies.

Rabies is caused by infection with the rabies virus: an enveloped, single-stranded RNA virus in the Lyssavirus genus and Rhab- doviridae family. ${ }^{6}$ The virus infects the peripheral nervous system of mammals and undergoes retrograde axonal transport to the central nervous system (CNS). ${ }^{7}$ Once the virus reaches the CNS it is universally fatal. From the CNS it spreads to multiple organs along both sensory and autonomic nerves. ${ }^{7}$ The disease has been feared globally for over 4000 years. ${ }^{6}$ It is the only virus known that has an acute case fatality rate of nearly $100 \%$, making it among the most deadly viruses on Earth. ${ }^{6-7}$ The World Health Organization estimates that rabies results in more than 50000 deaths annually., ${ }^{6,8}$ Most of these deaths occur in the developing world, especially in Asia and Africa, where dogs are not routinely immunized against the virus ${ }^{7}$ and PEP is not easily available or affordable for dog bite victims. ${ }^{6}$ In North America most cases of rabies occur due to bat bites. ${ }^{7}$ In the United States, there are fewer than 2 deaths annually from rabies. ${ }^{8}$ Once a patient has developed signs and symptoms of rabies, there are no effective measures to stop the progression of the disease. Timely rabies PEP is essential to preserve the life of a patient after a bite from a rabid animal. ${ }^{7}$

The incubation period of the virus in humans is typically 20 to 90 days. $^{7}$ The prodromal symptoms are non-specific but may include early localized symptoms of pain, itching and paresthesia at the bite site. ${ }^{7}$ There are two presentations of the infection: encephalitic rabies and paralytic rabies. ${ }^{6}$ The encephalitic form of the disease occurs in approximately $80 \%$ of human rabies cases. ${ }^{7}$ It is characterized by episodes of excitement and hallucinations with intermittent lucidity. Patients also have episodes of autonomic dysfunction and hydrophobia (fear of water). Death usually occurs within 7 days. ${ }^{7}$ In the paralytic form of the disease, patients develop quadriparesis, flaccid muscle weakness and sphincter dysfunction. These patients are deceased within 2 weeks. ${ }^{7}$

Dogs infected by the rabies virus excrete the virus in the saliva. ${ }^{4}$ A bite is an effective method of inoculating a victim with the virus. In an animal, signs of infection include lethargy, fever, vomiting, anorexia, cerebral dysfunction, cranial nerve dysfunction, ataxia, weakness, paralysis, seizures, abnormal behaviour, aggression and self-mutilation. ${ }^{4}$

On her first visit to the clinic, day 0, our 3-year-old patient received a shot of HyperRAB human anti-rabies immunoglobulin near the site of her bite and a shot of Imovax rabies vaccine at a site distant from her bite. The HyperRAB, a pooled immunoglobulin prepared from human donors with circulating anti-rabies anti- 


\section{CASE REPORT}

bodies, provides passive immunity against the rabies virus for up to 3 weeks following administration. ${ }^{8}$ Timely treatment with HyperRAB provides passive immunity to rabies before active immunity is acquired from vaccination with Imovax. ${ }^{8}$ Our patient received a second shot of Imovax vaccine on day 3 and a third shot on day 7 postexposure. Once the puppy had been quarantined for 10 days and was still alive and healthy and had tested negative for the rabies virus, our patient did not require the fourth injection of Imovax vaccine normally administered on day 14 . A fifth dose of vaccine is administered on day 28 in patients who are immunosuppressed. ${ }^{6,8}$ The side effects associated with HyperRAB and Imovax include local reactions at the site of injection such as erythema, itching and swelling, as well as systemic reactions such as headache, nausea, abdominal pain, myalgia, dizziness and fever. ${ }^{8-9}$ These are similar to side effects seen with other vaccines. Patients bitten by an animal should also receive a tetanus shot if their immunization status is not up-to-date. ${ }^{1}$ Our 3-year-old patient had received all of her scheduled tetanus vaccinations. If she were under 3 years of age, she would have required a tetanus vaccine on day 0 .

Our patient was one of the estimated 15 million people worldwide who receive PEP annually. ${ }^{9}$ The wound on her ear healed well and will not result in any disfigurement. At our last encounter, she told me that despite being bitten by a puppy she was not afraid of doggies. She then showed me how to gently pat doggies using her well-loved stuffed black dog. She had been practising.

\section{REFERENCES}

1. Sabhaney V, Goldman RD. Child health update. Management of dog bites in children. Can Fam Physician. 2012 Oct;58(10):1094-6.

2. Raghavan M. Fatal dog attacks in Canada, 1990-2007. Can Vet J. 2008 Jun;49(6):577-81.

3. Eppley BL, Schleich AR. Facial dog bite injuries in children: treatment and outcome assessment. J Craniofac Surg. 2013 Mar;24(2):384-6.

4. Centers for Disease Control and Prevention [Internet]. Atlanta: Centers for Disease Control and Prevention; c2004. Rabies Resource Page; [reviewed 2013 Mar 15; updated 2013 Sep 24; cited 2013 May 29]. Available from: www.cdc.gov/rabies

5. Ontario Veterinary Medicine Association. A Dog Owner's Handbook [Internet]. Milton (ON): Ontario Veterniary Medicine Association; 2013 [cited 2013 May 29]. Available from: publications.ovma.org/i/115942

6. Both L, Banyard AC, van Dolleweerd C, Horton DL, Ma JK, Fooks AR. Passive immunity in the prevention of rabies. Lancet Infect Dis. 2012 May;12(5):397-407.

7. Jackson AC. Current and future approaches to the therapy of human rabies. Antiviral Res. 2013 Jul;99(1):61-7.

8. Bailey AM, Holder MC, Baker SN, Weant KA. Rabies prophylaxis in the emergency department. Adv Emerg Nurs J. 2013 Apr-Jun;35(2):110-9; quiz 20-1.

9. Warrell MJ. Current rabies vaccines and prophylaxis schedules: preventing rabies before and after exposure. Travel Med Infect Dis. 2012 Jan;10(1):1-15. 